\title{
One-Step Covalent Surface Modification to Achieve Oil-Water Separation Performance of a Non-Fluorinated Durable Superhydrophobic Fabric
}

\author{
Mengmeng Zhou, Meng Li, Fengjiao Xu, Yuanping Yang, Yongbing Pei,* Yue Yan, and Lianbin $\mathrm{Wu}^{*}$ \\ Cite This: ACS Omega 2021, 6, 24139-24146 \\ Read Online
}

ABSTRACT: In this work, a durable superhydrophobic fabric was fabricated by a facile covalent surface modification strategy, in which the anchoring of 10undecenoyl chloride (UC) onto the fabric through the esterification reaction and covalent grafting of $n$-dodecyl-thiol (DT) via thiol-ene click chemistry were integrated into one step. Fourier transform infrared spectroscopy (FTIR), X-ray photoelectron spectroscopy (XPS), and scanning electron microscopy (SEM) measurement results demonstrated that UC and DT were covalently grafted onto the fabric surface. The formed gully-like rough structure by the grafted UC and DT on the fabric surface together with the inherent microfiber structure, combined with the grafted low-surface-energy materials of UC and DT, gave the resultant modified DT-UC@fabric superhydrophobic performance. The superhydrophobic DT-UC@ fabric was used for separation of oil-water mixtures; it exhibited high separation efficiency of more than $98 \%$. In addition, it presented excellent durability against mechanical damage; even after 100 cyclic tape-peeling and abrasion tests, the DT-UC@fabric could preserve superhydrophobic performance, which was ascribed to the formed covalent interactions between the fabric surface and the grafted UC and DT. Therefore, this work provided a facile, efficient strategy for fabricating superhydrophobic composites with excellent durability, which exhibited a promising prospect in the application of self-cleaning and oil-water separation.

\section{INTRODUCTION}

arge amount of oily wastewater produced by frequent oil spills and leaks of organic compounds is one of the significant threats to human's living environment and health, ${ }^{1,2}$ which impels the development of novel approaches and materials for efficient oil-water separation, considered to be a great issue of concern. ${ }^{3,4}$ A variety of techniques and materials have been employed for cleaning oil-contaminated water, including physical absorption, ${ }^{5}$ in situ burning, ${ }^{6}$ bioremediation, ${ }^{7}$ etc. However, these traditional cleanup methods consume substantial time and human, material, and financial resources and have low efficiencies. Since special superhydrophobic/ superoleophilic materials were reported for oil-water separation, ${ }^{8}$ diverse types of superhydrophobic/superoleophilic materials including porous sponges ${ }^{9,10}$ and fabrics ${ }^{11,12}$ were developed. Particularly, cellulose-based fabrics like cotton and woven have gained tremendous attention owing to their superiority in the aspects of light weight, low cost, recycling, biodegradability, and excellent mechanical strength. However, the fabric surface needs to be modified to achieve superhydrophobic properties prior to application. Recently, various strategies including dip-coating, ${ }^{13}$ chemical vapor deposition, ${ }^{14}$ and plasma treatment ${ }^{15}$ have been developed to fabricate superhydrophobic fabrics. These methods commonly involve depositing nanoparticles or low-surface-energy substances onto the fabric surface. Unfortunately, superhydrophobic surfaces are vulnerable to mechanical damage. To enhance durability, it is common to establish covalent bonds between fabrics and nanoparticles or low-surface-energy substances. ${ }^{16}$

Up to date, as a novel chemical modification method, photoinduced thiol-ene click chemistry has been applied to prepare superhydrophobic fabrics owing to its highly efficient covalent grafting of ene-terminated molecules on thiol-terminated surfaces under UV light. ${ }^{17-19}$ For instance, Hou et al. grafted methacryl-heptaisobutyl polyhedral oligomeric silsesquioxane (MA-POSS) onto a sulfhydryl pretreated cotton fabric via thiol-ene click chemistry. The formed rough structure and low surface energy of MA-POSS promoted fabric superhydrophobicity. ${ }^{20}$ Xue et al. used a similar method to graft dodecafluoroheptyl methacrylate onto sulfhydryl-pretreated fabrics to obtain superhydrophobic fabrics. ${ }^{21}$ These methods provide the fabric excellent mechanical durability arising from the covalent bond interaction between fabrics and low-surface-

Received: July 10, 2021

Published: September 8, 2021 


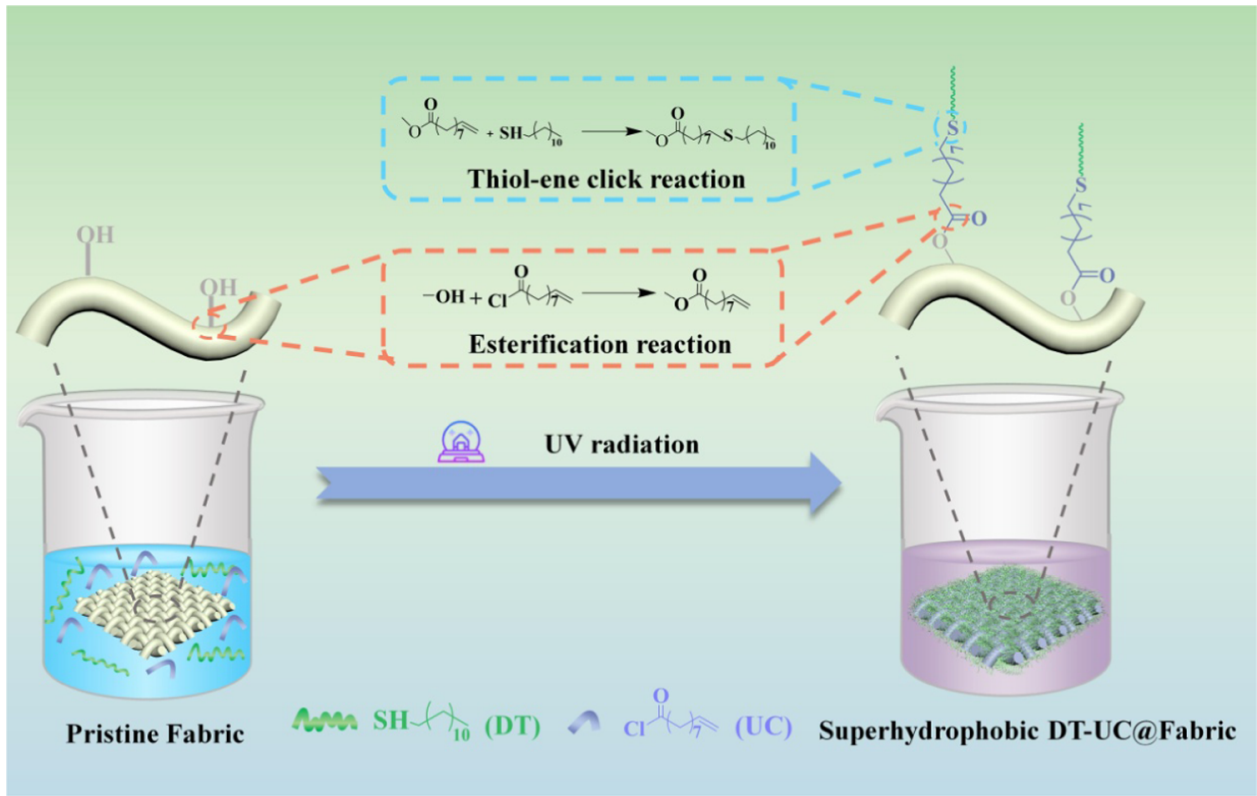

Figure 1. Schematic preparation of a superhydrophobic DT-UC@fabric.

energy compounds. However, these methods suffer from cumbersome steps, such as alkali etching or sulfhydryl treatment on the fabric in advance, as well as involvement of environment unfriendly low-surface-energy substances like fluoride.

Inspired by previous studies, we present a facile, one-step, fluorine-free highly efficient strategy to prepare a durable superhydrophobic fabric, which is schematically illustrated in Figure 1. The anchoring of high chemical activity of 10undecenoyl chloride (UC) onto fabrics through the esterification reaction and covalent grafting of $n$-dodecyl-thiol (DT) via thiol-ene click chemistry are combined into one step with the aid of UV light. The bridging effect of UC in the reaction process ensures the optimum attachment of DT on the fabric surface. The combination of the formed rough structure and low-surface-energy materials promoted the fabric to be superhydrophobic and could be used for oil-water separation. More interestingly, the as-prepared fabric exhibited excellent durability against mechanical damages like abrasion and tape peeling. This was associated with the strong covalent interactions between the grafted UC and DT and fabric fibers. Hence, this work provided a facile, high-efficiency strategy to fabricate a durable superhydrophobic fabric, exhibiting promising application in the field of oil recovery and water purification.

\section{RESULTS AND DISCUSSION}

2.1. Preparation of the Superhydophobic DT-UC@ Fabric. In the current work, a cellulous fabric was used as the starting material. A facile one-step dip-coating strategy was developed to prepare a superhydrophobic fabric, which is schematically illustrated in Figure 1. The cellulose-based fabric was immersed into a solution containing 10-undecenoyl chloride (UC), $n$-dodecylthiol (DT), and the photo-initiator HMPF. The naturally existent hydroxyl groups in the fabric readily converted to esters by the treatment of the fabric with $\mathrm{UC}^{22}$ thus, UC was grafted onto the fabric surface through the esterification reaction with hydroxyl groups due to its high chemical activity. Accompanying with the esterification reaction, the thiol-ene click reaction also occurred between ene-containing UC and thiol-containing DT with the assistance of UV irradiation with a wavelength of $365 \mathrm{~nm}$, resulting in DT molecules covalently anchored onto the fabric surface. Upon the completion of the reaction, the fabric was washed with water, ethanol, and acetone to remove impurities followed by drying in an oven, and the target fabric was obtained (designated as DT-UC@fabric).

The key parameters affecting the fabric surface including UC and DT concentrations were optimized to get the best characteristics. Considering thiol-ene click chemistry is a highly efficient reaction, in which the theoretical molar ratio of thiol and ene groups is 1:1, the molar ratio of UC and DT was fixed at $1: 1$. To investigate the effect of UC and DT concentrations on the hydrophobicity of the DT-UC@fabric, the mass concentration of UC was adopted for convenience, and the corresponding results are plotted in Figure 2a. When the UC concentration was increased from 2 to $8 \%$, the water contact angle (WCA) of the DT-UC@fabric increased from 135 to $156^{\circ}$, indicating that the DT-UC@fabric was superhydrophobic. Afterward, the WCA reached the plateau at $156^{\circ}$ even when the UC concentration was further increased to $10 \%$. This might be explained as follows. When the UC concentration reached $8 \%$, all hydroxyl groups on the fabric surface were converted to ester groups completely and the UC loading amount reached a maximum. The further increase of the UC concentration could not bring about an increase in the UC loading amount; thus, the WCA did not increase. Therefore, the UC concentration was optimized as $8 \%$, and the corresponding fabric was utilized for further discussion.

The FTIR spectra of UC, DT, pristine, and DT-UC@ fabrics are shown in Figure $2 \mathrm{~b}$. Compared with the pristine fabric, two evident peaks were observed in the DT-UC@ fabric. First, the stretching vibration peaks of $\mathrm{C}-\mathrm{H}$ at 2920 and $2896 \mathrm{~cm}^{-123}$ in the DT-UC@fabric are attributed to the alkyl groups in UC and DT. Furthermore, the peaks at 1626 and $2540 \mathrm{~cm}^{-1}$ observed in the spectra of UC and DT are attributed to $\mathrm{C}=\mathrm{C}^{24}$ and $\mathrm{S}-\mathrm{H},{ }^{25}$ respectively. However, these two peaks were not detected in the DT-UC@fabric, 

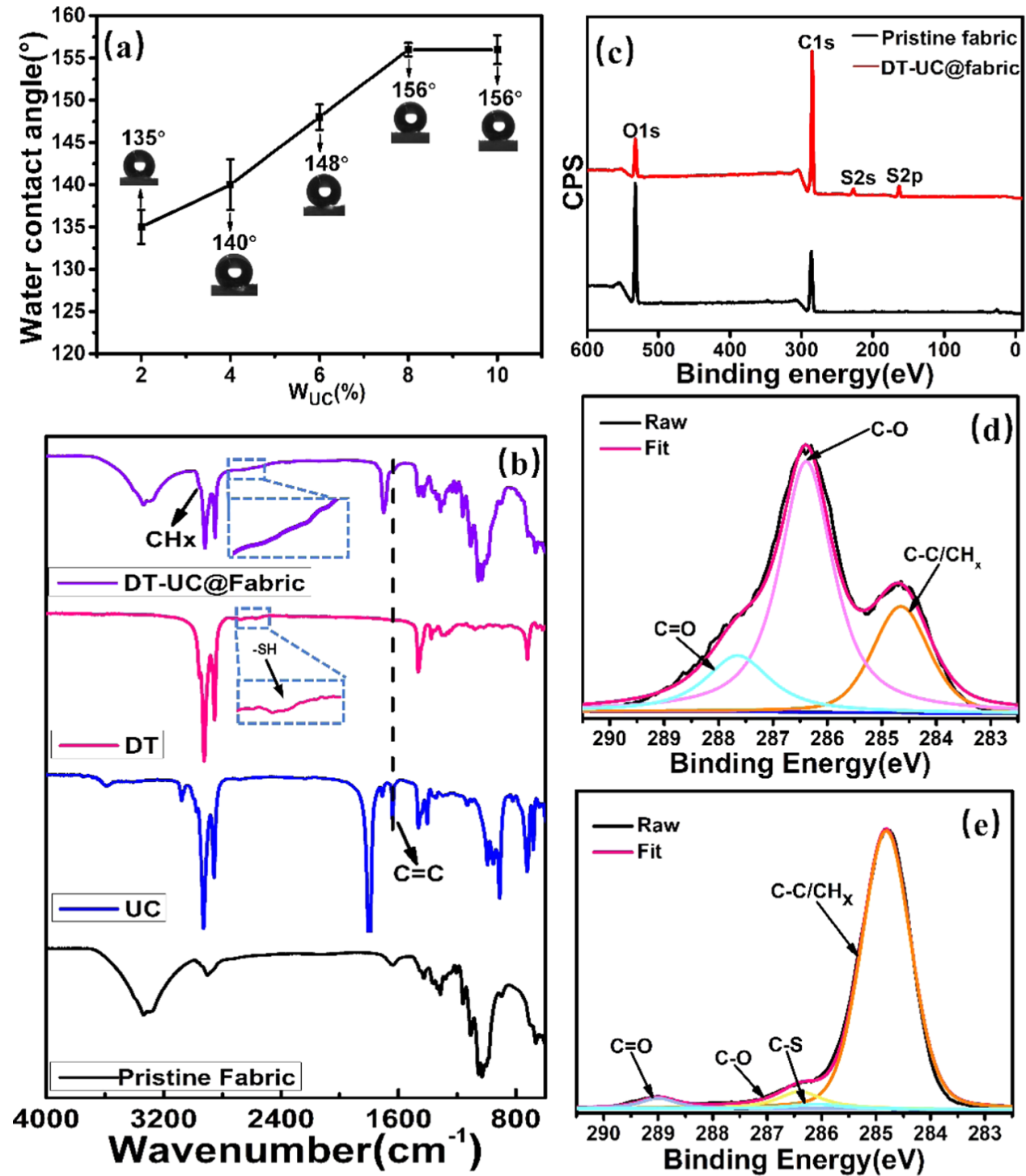

Figure 2. (a) Effect of the UC concentration on hydrophobicity of the DT-UC@fabric. (b) FTIR and (c) XPS spectra of pristine and DT-UC@ fabrics. High-resolution C 1s spectra of (d) pristine and (e) DT-UC@fabrics.

indicating that the $\mathrm{C}=\mathrm{C}$ and $\mathrm{S}-\mathrm{H}$ bonds had undergone the thiol-ene click reaction. To further understand the chemical evolution before and after modification, XPS was employed to monitor the fabric surface, and the XPS survey spectrum is shown in Figure 2c. The pristine fabric was only composed of $\mathrm{C}$ and $\mathrm{O}$ elements, with the $\mathrm{C} 1 \mathrm{~s}$ peak and $\mathrm{O} 1 \mathrm{~s}$ peak at binding energies of 533 and $285 \mathrm{eV}^{26}$ respectively. However, for the DT-UC@fabric, besides the original C and O peaks, new peaks of $S 2 p$ and $S 2 s$ were also detected at binding energies of 166.6 and $230.1 \mathrm{eV},{ }^{19,21}$ which could be from the $S$ element in DT. Furthermore, in their high-resolution $\mathrm{C}$ 1s spectra, three obvious peaks located at $287.6 \mathrm{eV}(\mathrm{C}=\mathrm{O})$, $286.4 \mathrm{eV}(\mathrm{C}-\mathrm{O})$, and $284.6 \mathrm{eV}(\mathrm{C}-\mathrm{C})$ were observed in the pristine fabric (Figure $2 \mathrm{~d}$ ), while a new peak was detected at $286.1 \mathrm{eV}$ in Figure 2e, which should be ascribed to the $\mathrm{C}-\mathrm{S}$ bond. ${ }^{27}$ Moreover, the $\mathrm{C}-\mathrm{O}$ peak was weakened, while the $\mathrm{C}-$ $\mathrm{C}$ peak was significantly increased in Figure 2e compared with the pristine fabric, indicating that the surface of DT-UC@ fabric was dominated by a large amount of alkyl groups. This further verified that UC and DT had grafted onto the fabric surface through the one-step covalent surface modification method.

2.2. Surface Analysis of the Superhydophobic DTUC@Fabric. The surface morphologies of pristine and DTUC@fabrics are characterized by SEM and shown in Figure 3a-f, respectively. Both the fibers in pristine and DT-UC@ fabrics are in ordered arrangement (see Figure $3 a, d$ ), but it should to be noted that the surface on the pristine fabric is very smooth except some inherent texture (see Figure $3 b, c$ ) due to the wicking effect of the porous structure, ${ }^{28,29}$ whereas the surface on the DT-UC@fabric is pretty rough, where tortuous gully-like structures are clearly observed on the microfiber surface (see Figure 3e,f).

Besides the SEM images, the surface morphologies of pristine and modified fabrics are further characterized by AFM, as shown in Figure $3 g-1$, respectively. Compared with the pristine fabric, the $3 \mathrm{D}$ surface morphology of the modified DT-UC@fabric became rougher than that of the pristine fabric (see Figure 3g,j), the root mean square roughness ( $\mathrm{Rq}$ ) of the fabric increasure from 14.649 to $149.856 \mathrm{~nm}$, and the height difference was increased from 51.81 to $351.95 \mathrm{~nm}$ from 


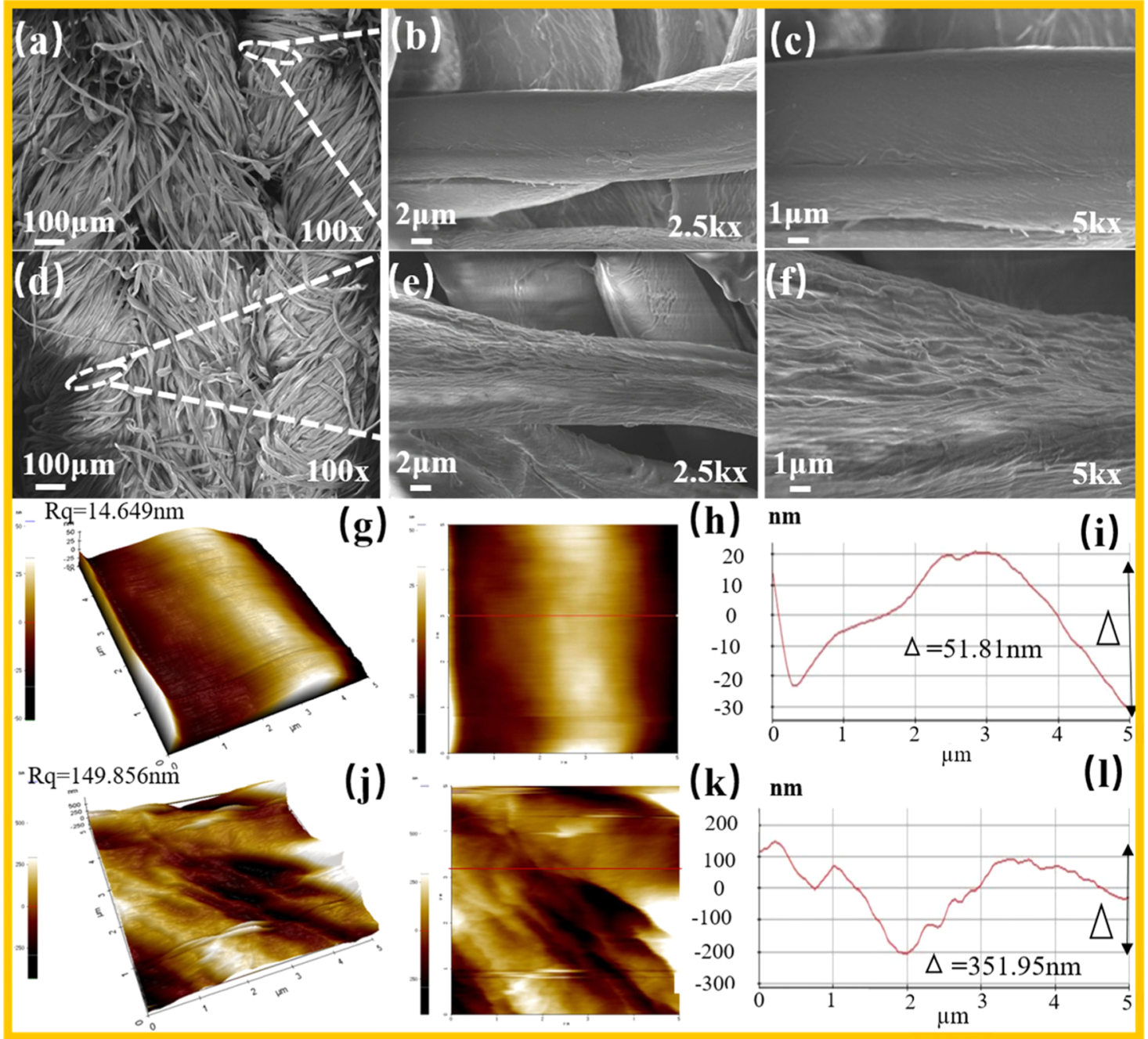

Figure 3. SEM images of $(a-c)$ the pristine fabric and $(d-f)$ the DT-UC@fabric. AFM images of $(g-i)$ the pristine fabric and ( -1$)$ the DTUC@fabric.

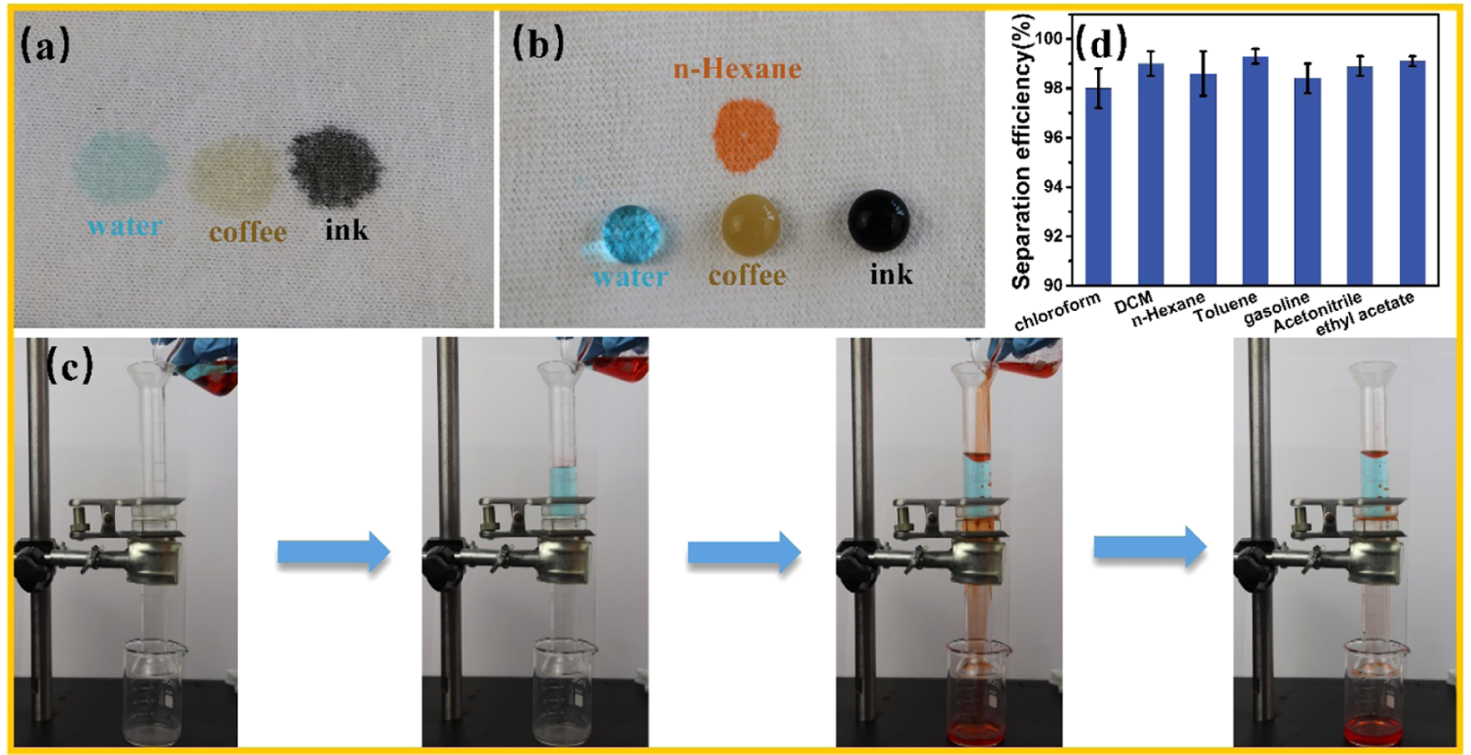

Figure 4. Digital photographs of water, coffee, and ink droplets on (a) pristine and (b) DT-UC@fabrics. (c) Separation process of the dichloromethane/water mixture (DCM was dyed with Sudan II, water was dyed with $\mathrm{CuSO}_{4}$ ). (d) Separation efficiency of the DT-UC@fabric for various organic solvent/water mixtures. 

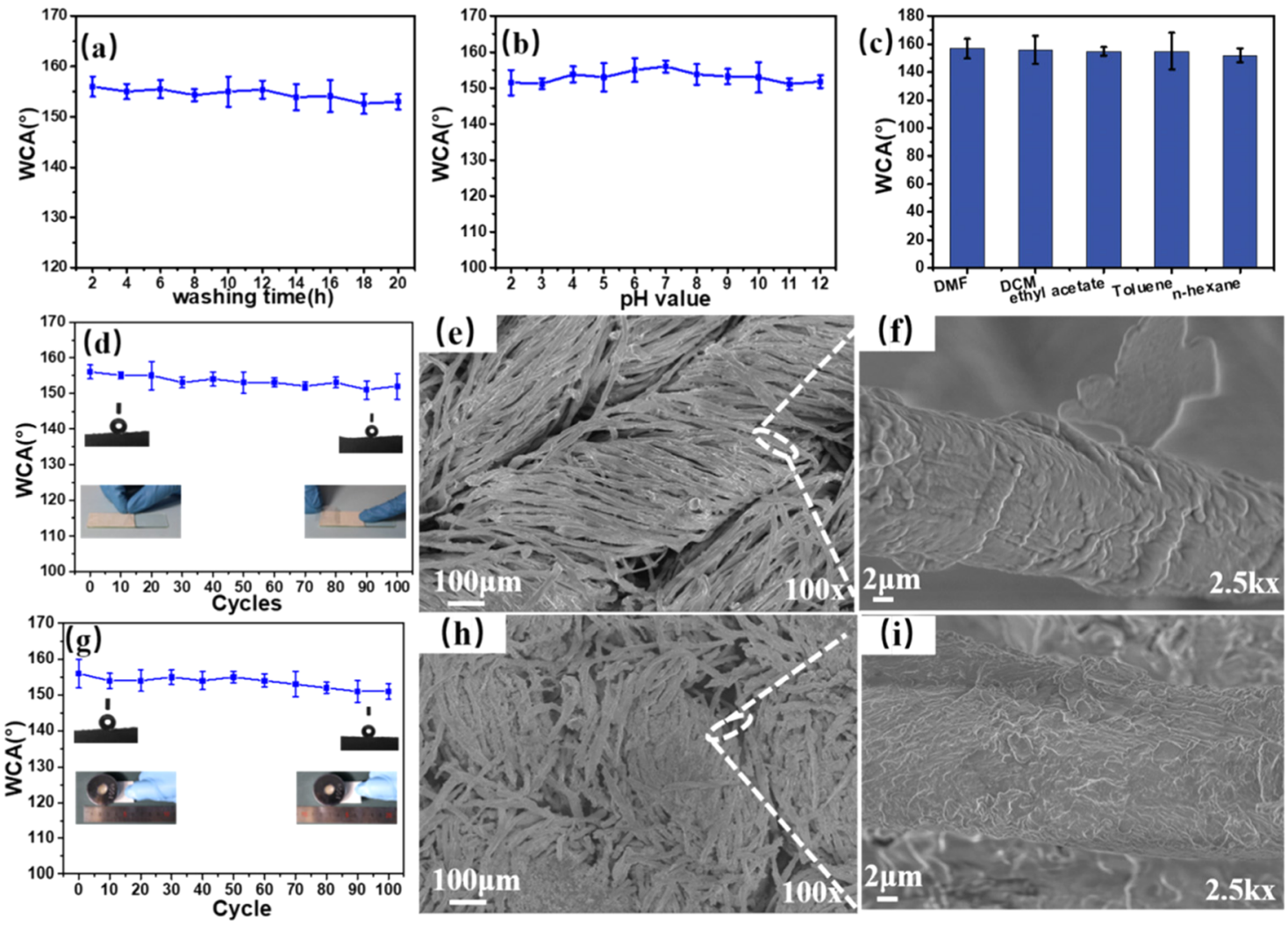

Figure 5. WCA variation of the DT-UC@fabric at (a) various washing times, (b) water droplets with various $\mathrm{pH}$ values, and (c) soaking with various organic solvents. (d) WCA variations and (e, f) SEM images of the DT-UC@fabric during the tape-peeling test. (g) WCA variations and (h, i) SEM images of the DT-UC@fabric during the abrasion test.

the analysis chart (see Figure 3i,l). The structural evolution on the fabric surface should be a result of the covalently grafted UC and DT during the one-step modification. More evidence was found in the EDS spectra and element mapping of the DT-UC@fabric (see Figure S1), in which new occurrences of element $\mathrm{S}$ was detected; meanwhile, the element content of $\mathrm{C}$ increased but the $\mathrm{O}$ element decreased after modification, which verified large amounts of long alkyl chains in UC and DT had grafted on the fabric surface, resulting in the fabric fiber become rough due to the wrinkle effect. ${ }^{30}$

2.3. Wetting Behavior and Oil-Water Separation Performance of the DT-UC@Fabric. As depicted in Figure $4 a, b$, the photographs recorded the sitting status of water, coffee, and ink droplets on pristine and DT-UC@fabrics, respectively. When these droplets were dropped onto the pristine fabric, they spread quickly and then were absorbed. While when water, coffee, and ink droplets were dropped onto the DT-UC@fabric surface, these droplets could stand and formed a round shape with WCA $\sim 156^{\circ}$ and maintained the same shape even after $30 \mathrm{~min}$, indicating that the DT-UC@ fabric featured stable superhydrophobic performance. While when $n$-hexane was dropped on the DT-UC@fabric surface, it was absorbed immediately. Additionally, when the DT-UC@ fabric was completely immersed into water, a mirror-like phenomenon was observed (Figure S2), where an air bubble layer was formed on the surface of the fabric. This phenomenon could be attributed to the entrapped air cushion between water and the modified fabric surface, which was formed due to the micro-nano hierarchical structure in which air molecules could retain. This air cushion acted as a shield to effectively inhibit water penetration into the fabric surface even in full immersion. After being taken out of water, the surface of the fabric was not wet, implying that the as-prepared DTUC@fabric displayed durable superhydrophobicity. Furthermore, the superhydrophobic DT-UC@fabric presented a selfcleaning effect when it was contaminated with dust. When water droplets were continuously dropped onto the tilted fabric surface, they would roll off the surface at once and flush away the dust layer, and the dust-contaminated fabric would return to be clean (Figure S3 and Video S1). Based on its special oil-absorbing water-repelling feature, it could absorb dichloromethane and remove them from the dichloromethane/water mixture (Figure S4 and Video S2). Considering its inherent high porosity, the superhydrophobic DTUC@fabric could be utilized for continuous separation of oilwater mixture. A simple equipment was set up in Figure 4c, where the superhydrophobic DT-UC@fabric was sandwiched between two double-pass pipes. Pouring the dichloromethane/ water mixture into the double-pass pipe, dichloromethane would settle to the bottom due to its large density. Once dichloromethane approached the DT-UC@fabric, it was absorbed immediately due to the superoleophilicity, which could be owing to the tortuous gully-like structures and the grafted hydrophobic long alkyl chain; thus, a layer of dichloromethane was formed on the fabric, which increased the contact area with oil droplets. This was based on the theoretical intrusion pressure formula as follows ${ }^{31,32}$

$$
\Delta p=\frac{2 \gamma}{R}=-\frac{L \gamma \cos \theta}{A}
$$

where $\gamma$ is the surface tension, $R$ is the radius of the meniscus, and $L$ and $A$ are the circumference and the area of the fiber 
pore, respectively. $\theta$ is the advancing angle on the fabric. According to this equation, it can be seen that for oil, the fabric cannot bear any pressure due to $\Delta p<0$; thus, oil can easily pass through the fabric, while the water can be supported because $\Delta p>0$. As time elapsed, the absorbed dichloromethane gradually penetrated into the fabric and flows slowly into the beaker under gravity, while water remained on the upper layer throughout the process (see Video S3). As a result, dichloromethane was successfully separated from the dichloromethane/water mixture. Moreover, separation efficiency was taken into account to evaluate its separation performance. The separation efficiency of the DT-UC@fabric for chloroform, dichloromethane, hexane, toluene, gasoline, acetonitrile, and ethyl acetate was above $98 \%$ in every case, as shown in Figure $4 \mathrm{~d}$, indicating the DT-UC@fabric possessed excellent separation performance.

2.4. Durability of the Superhydrophobic DT-UC@ Fabric. In general, most superhydrophobic surfaces feature poor durability and are vulnerable to mechanical damage, corrosive solution environment, and high-temperature exposure; thus, it is essential to investigate their durability under different conditions. ${ }^{33-36}$ Here, the durability of the prepared superhydrophobic DT-UC@fabric was evaluated by water washing, as well as acid and alkaline conditions and organic solvent exposures. For the stability of washing tests, the fabric's WCA can be maintained over $150^{\circ}$ even with washing that lasts for $20 \mathrm{~h}$, as shown in Figure 5a. When encountering acid and alkaline water droplets in a wide $\mathrm{pH}$ range $(2-12)$, the fabric surface can preserve its superhydrophobic property, as shown in Figure 5b. Additionally, even after a long time of immersion in an organic reagent, the fabric does not lose its superhydrophobic performance, as shown in Figure 5c.

Furthermore, the durability of the DT-UC@fabric against mechanical damage was evaluated by tape-peeling and abrasion tests. The effect of tape-peeling times on WCA is shown in Figure 5d. The WCA of the DT-UC@fabric only decreased slightly with the increase in tape-peeling tests; even when tapepeeling cycles increased to 100 , the fabric still preserved its superhydrophobicity (WCA about $152^{\circ}$ ). Figure 5 e,f revealed the SEM images of DT-UC@fabric after the tape-peeling test. When compared with the sample before the test, the overall fibers remained in a similar state, except for becoming floating and loose, not compact as before (see Figures 5e and $3 \mathrm{~d}$ ). In the magnified SEM images, no obvious destruction of the rough structure was observed, but minor changes like tortuous gully-like structures disappeared (see Figures $5 \mathrm{f}$ and $3 \mathrm{e}$ ). This could be due to the adhesive effect of the tape, which also makes the low-surface-energy materials leave the fiber surface; herein, the WCA of the DT-UC@fabric decreased slightly but remained larger than $150^{\circ}$.

The influence of abrasion on WCA variations of DT-UC@ fabric is shown in Figure $5 \mathrm{~g}$. With the increase in cyclic abrasion, the WCA of the DT-UC@fabric showed a trend of slight decrease but remained about $151^{\circ}$ after 100 abrasion cycles. Figure $5 \mathrm{~h}$ showed that the overall morphology of the DT-UC@fabric surface did not change significantly; only the fiber arrangement and distribution became more compact compared with that before test (see Figure 3d), which should be associated with the abrasion pressure. In a magnified image (see Figure 5i), the fiber surface structure remained clearly rough. One should note that the previous tortuous gully-like structures transformed into a wrinkled state, implying some grafted materials were desquamated from the fabric surface, thus leading to a slight decrease in WCA. The abovementioned tests indicated the DT-UC@fabric had excellent mechanical durability against damage; this should be derived from the formation of strong chemical bond between UC and DT and the fabric.

\section{CONCLUSIONS}

A non-fluorinated durable superhydrophobic DT-UC@fabric was prepared via one-step covalent surface modification. It exhibited high oil-water separation efficiency of larger than $98 \%$ for various organic solvent/water mixtures. Meanwhile, the DT-UC@fabric presented excellent durability and could preserve superhydrophobic performance against mechanical damage. This was associated with strong covalent interactions between the grafted UC and DT and fabric fibers by the onestep surface modification method. Hence, this work provides a facile, high-efficiency strategy to fabricate a durable superhydrophobic fabric.

\section{EXPERIMENTAL SECTION}

4.1. Materials. A cellulose-based fabric was obtained from the local supermarket, 10-undecenoyl chloride (UC) was obtained from Aladdin Chemistry Co. Ltd., n-dodecylthiol (DT) was provided by Jiuding Chemical Technology Co., Ltd. (Shanghai, China), and 2-hydroxy-2-methylpropiophenone (HMPF) was purchased from Aladdin Chemistry Co., Ltd. (China). Dichloromethane (DCM) and other solvents were purchased from Sinopharm Chemical Reagent Co., Ltd. (Shanghai, China). All reagents were of analytical grade and directly used.

4.2. One-Step Preparation of the Superhydrophobic DT-UC@Fabric. The preparation formula of a DT-UC@ fabric is shown in Table 1. First, pieces of the pristine cellulose

Table 1. Experimental Formula for the Preparation of the DT-UC@Fabric

\begin{tabular}{ccccc} 
UC mass concentration (\%) & UC $(\mathrm{g})$ & DT $(\mathrm{g})$ & DCM $(\mathrm{g})$ & HMPF $(\mathrm{g})$ \\
2 & 0.8 & 0.8 & 40 & 0.2 \\
4 & 1.6 & 1.6 & 40 & 0.2 \\
6 & 2.4 & 2.4 & 40 & 0.2 \\
8 & 3.2 & 3.2 & 40 & 0.2 \\
10 & 4.0 & 4.0 & 40 & 0.2 \\
\hline
\end{tabular}

fabric $(2 \mathrm{~cm} \times 2 \mathrm{~cm})$ were washed with ethanol several times under sonication to remove surface impurities and then dried at $60{ }^{\circ} \mathrm{C}$. Typically, the cleaned fabric was immersed into the mixture solution of $40 \mathrm{~g}$ of dichloromethane, $0.2 \mathrm{~g}$ of HMPF, $3.2 \mathrm{~g} \mathrm{DT}$, and $3.2 \mathrm{~g}$ of $\mathrm{UC}$, and then the solution containing fabric was exposed to UV light with an intensity of $300 \mathrm{~W} \mathrm{~m}^{-2}$ at $365 \mathrm{~nm}$ from the top side for $30 \mathrm{~min}$ under vigorous magnetic stirring at room temperature. Finally, the cellulosebased fabric was taken out and washed successively with dichloromethane and then was dried at $60{ }^{\circ} \mathrm{C}$ in an oven. The resultant fabric was denoted DT-UC@fabric.

4.3. Characterization. Fourier transform infrared (FTIR) spectra were recorded on a spectroscope (Antaris, Nicolet 7000 ) in the range from 4000 to $400 \mathrm{~cm}^{-1}$. High-resolution Xray photoelectron spectroscopy (XPS) measurements were performed using VG Scientific ESCALab 220I-XL equipped with a $\mathrm{Mg} \mathrm{K} \alpha$ X-ray source and a hemispherical electron analyzer. The morphology of the fabric was observed using a scanning electron microscope (SEM, Zeiss ultra plus) and an 
atomic force microscope (AFM, Park Systems NX10). The surface hydrophobicity was measured using a DSA30 contact angle analyzer (KRUSS, Germany).

4.4. Oil-Water Separation Efficiency and Durability Evaluation. A DT-UC@fabric was used to separate a mixture solution of $100 \mathrm{~mL}$ of water and 100 of $\mathrm{mL}$ solvent. Once the separation was completed, the separated solvents were collected, and the separation efficiency $(E)$ was calculated with the following formula

$$
E=V / 100 \times 100 \%
$$

where $V$ represents the volume of collected solvents after separation from the mixture.

For testing the durability with washing tests, the fabric's WCA was tested after various washing times, and the longest washing time was $20 \mathrm{~h}$. For testing the stability against acid and alkali resistance, water droplets in the $\mathrm{pH}$ range $2-12$ were dropped on the fabric surface, and the fabric's WCA was tested at least $1 \mathrm{~min}$ later in a stable state. For testing the stability the organic reagent resistance, the fabric was immersed into the organic reagents for $24 \mathrm{~h}$, and then, the fabric's WCA was tested after drying.

For the durability against tape peeling, the DT-UC@fabric was fixed on a glass slide by a double-sided tape; a $30 \mathrm{kPa}$ pressure was exerted to achieve a good contact between the fabric surface and the tape. The tape-peeling process starting from pressing and ending as peeling-off the tape was designated as one cycle. A total of 100 cycles were conducted, and WCA was measured every 10 cycles.

For the durability against abrasion, the DT-UC@fabric was placed on a sandpaper (300 mesh), and then, it was moved forward $10 \mathrm{~cm}$ at a rate of $20 \mathrm{~cm} \mathrm{~min}{ }^{-1}$ under external force accompanied with a $100 \mathrm{~g}$ balancing weight. This process was defined as one cycle. A total of 100 cycles were conducted, and WCA was measured every 10 cycles.

\section{ASSOCIATED CONTENT}

\section{SI Supporting Information}

The Supporting Information is available free of charge at https://pubs.acs.org/doi/10.1021/acsomega.1c03642.

EDS spectra and element mapping of pristine and DTUC@fabrics (Figure S1); mirror-like phenomenon of the DT-UC@fabric (Figure S2); self-cleaning process of the DT-UC@fabric (Figure S3); and removal process of dichloromethane from the dichloromethane/water mixture (Figure S4) (PDF)

Self-cleaning process of the DT-UC@fabric contaminated by a layer of dust (AVI)

The removal of dichloromethane droplet from dichloromethane/water mixture (AVI)

Separation process of dichloromethane/water mixture (AVI)

\section{AUTHOR INFORMATION}

\section{Corresponding Authors}

Yongbing Pei - Key Laboratory of Organosilicon Chemistry and Materials Technology, Ministry of Education, Hangzhou Normal University, Hangzhou 311121, P. R. China; (1) orcid.org/0000-0003-3006-1317; Email: peiyongbing@ hznu.edu.cn

Lianbin Wu - Key Laboratory of Organosilicon Chemistry and Materials Technology, Ministry of Education, Hangzhou
Normal University, Hangzhou 311121, P. R. China;

○ orcid.org/0000-0003-3403-2142; Email: wulianbin@ hznu.edu.cn

\section{Authors}

Mengmeng Zhou - Key Laboratory of Organosilicon Chemistry and Materials Technology, Ministry of Education, Hangzhou Normal University, Hangzhou 311121, P. R. China

Meng Li - Key Laboratory of Organosilicon Chemistry and Materials Technology, Ministry of Education, Hangzhou Normal University, Hangzhou 311121, P. R. China

Fengjiao Xu - Key Laboratory of Organosilicon Chemistry and Materials Technology, Ministry of Education, Hangzhou Normal University, Hangzhou 311121, P. R. China

Yuanping Yang - Key Laboratory of Organosilicon Chemistry and Materials Technology, Ministry of Education, Hangzhou Normal University, Hangzhou 311121, P. R. China

Yue Yan - Key Laboratory of Organosilicon Chemistry and Materials Technology, Ministry of Education, Hangzhou Normal University, Hangzhou 311121, P. R. China

Complete contact information is available at: https://pubs.acs.org/10.1021/acsomega.1c03642

\section{Notes}

The authors declare no competing financial interest.

\section{ACKNOWLEDGMENTS}

This work was supported by the National Key Research and Development Program (2017YFB0307700) and the Department of Scientific and Technology of Zhejiang Province (LGG19E030007, LGG18E030007). The authors also thank the financial support from the Department of Scientific and Technology of Yunnan Province (202002AB080002).

\section{REFERENCES}

(1) Chu, Z.; Feng, Y.; Seeger, S. Oil/water separation with selective superantiwetting/superwetting surface materials. Angew. Chem., Int. Ed. 2015, 54, 2328-2338.

(2) Yu, Z.-P.; Zhan, B.; Dong, L.-M.; Jiang, W.; Song, Y.-Y.; Hu, S.A. Self-Healing Structured Graphene Surface with Reversible Wettability for Oil-Water Separation. ACS Appl. Nano Mater. 2019, 2, 1505-1515.

(3) Zhang, C.; Li, P.; Cao, B. Fabrication of SuperhydrophobicSuperoleophilic Fabrics by an Etching and Dip-Coating Two-Step Method for Oil-Water Separation. Ind. Eng. Chem. Res. 2016, 55, 5030-5035.

(4) Zhou, X.; Zhang, Z.; Xu, X.; Guo, F.; Zhu, X.; Men, X.; Ge, B. Robust and durable superhydrophobic cotton fabrics for oil/water separation. ACS Appl. Mater. Interfaces 2013, 5, 7208-7214.

(5) Calcagnile, P.; Fragouli, D.; Bayer, I. S.; Anyfantis, G. C.; Martiradonna, L.; Cozzoli, P. D.; Cingolani, R.; Athanassiou, A. J. A. n. Magnetically driven floating foams for the removal of oil contaminants from water. ACS Nano 2012, 6, 5413-5419.

(6) van Gelderen, L.; Malmquist, L. M.; Jomaas, G. J. F. Vaporization order and burning efficiency of crude oils during insitu burning on water. Fuel 2017, 191, 528-537.

(7) Gupta, V. K.; Carrott, P. J. M.; Ribeiro Carrott, M. M. L.; Suhas. Low-Cost Adsorbents: Growing Approach to Wastewater Treatment-a Review. Crit. Rev. Environ. Sci. Technol. 2009, 39, 783-842.

(8) Feng, L.; Zhang, Z.; Mai, Z.; Ma, Y.; Liu, B.; Jiang, L.; Zhu, D. A super-hydrophobic and super-oleophilic coating mesh film for the separation of oil and water. Angew. Chem., Int. Ed. 2004, 43, 20122014. 
(9) Liu, C.; Fang, Y.; Miao, X.; Pei, Y.; Yan, Y.; Xiao, W.; Wu, L. Facile fabrication of superhydrophobic polyurethane sponge towards oil-water separation with exceptional flame-retardant performance. Sep. Purif. Technol. 2019, 229, No. 115801.

(10) Lei, Z.; Zhang, G.; Deng, Y.; Wang, C. Thermoresponsive Melamine Sponges with Switchable Wettability by Interface-Initiated Atom Transfer Radical Polymerization for Oil/Water Separation. ACS Appl. Mater. Interfaces 2017, 9, 8967-8974.

(11) Jiang, C.; Liu, W.; Yang, M.; Liu, C.; He, S.; Xie, Y.; Wang, Z. Robust multifunctional superhydrophobic fabric with UV induced reversible wettability, photocatalytic self-cleaning property, and oilwater separation via thiol-ene click chemistry. Appl. Surf. Sci. 2019, $463,34-44$.

(12) Zeng, X.; Yang, K.; Huang, C.; Yang, K.; Xu, S.; Wang, L.; Pi, P.; Wen, X. Novel pH-Responsive Smart Fabric: From Switchable Wettability to Controllable On-Demand Oil/Water Separation. ACS Sustainable Chem. Eng. 2019, 7, 368-376.

(13) Deng, Y.; Han, D.; Deng, Y.-Y.; Zhang, Q.; Chen, F.; Fu, Q. Facile one-step preparation of robust hydrophobic cotton fabrics by covalent bonding polyhedral oligomeric silsesquioxane for ultrafast oil/water separation. Chem. Eng. J. 2020, 379, No. 122391.

(14) Feng, J.; Sun, M.; Ye, Y. Ultradurable underwater superoleophobic surfaces obtained by vapor-synthesized layered polymer nanocoatings for highly efficient oil-water separation. J. Mater. Chem. A 2017, 5, 14990-14995.

(15) Liu, S.; Zhou, H.; Wang, H.; Zhao, Y.; Shao, H.; Xu, Z.; Feng, Z.; Liu, D.; Lin, T. Argon Plasma Treatment of Fluorine-Free Silane Coatings: A Facile, Environment-Friendly Method to Prepare Durable, Superhydrophobic Fabrics. Adv. Mater. Interfaces 2017, 4, No. 1700027.

(16) Zhao, J.; Zhu, W.; Wang, X.; Liu, L.; Yu, J.; Ding, B. FluorineFree Waterborne Coating for Environmentally Friendly, Robustly Water-Resistant, and Highly Breathable Fibrous Textiles. ACS Nano 2020, 14, 1045-1054.

(17) Li, J.; Li, L.; Du, X.; Feng, W.; Welle, A.; Trapp, O.; Grunze, M.; Hirtz, M.; Levkin, P. A. Reactive superhydrophobic surface and its photoinduced disulfide-ene and thiol-ene (bio)functionalization. Nano Lett. 2015, 15, 675-681.

(18) Martin, K. L.; Nyquist, Y.; Burnett, E. K.; Briseno, A. L.; Carter, K. R. Surface Grafting of Functionalized Poly(thiophene)s Using Thiol-Ene Click Chemistry for Thin Film Stabilization. ACS Appl. Mater. Interfaces 2016, 8, 30543-30551.

(19) Fang, Y.; Liu, C.; Li, M.; Miao, X.; Pei, Y.; Yan, Y.; Xiao, W.; $\mathrm{Wu}, \mathrm{L}$. Facile Generation of Durable Superhydrophobic Fabrics toward Oil/Water Separation via Thiol-Ene Click Chemistry. Ind. Eng. Chem. Res. 2020, 59, 6130-6140.

(20) Hou, K.; Zeng, Y.; Zhou, C.; Chen, J.; Wen, X.; Xu, S.; Cheng, J.; Pi, P. Facile generation of robust POSS-based superhydrophobic fabrics via thiol-ene click chemistry. Chem. Eng. J. 2018, 332, 150159.

(21) Xue, C.-H.; Guo, X.-J.; Zhang, M.-M.; Ma, J.-Z.; Jia, S.-T. Fabrication of robust superhydrophobic surfaces by modification of chemically roughened fibers via thiol-ene click chemistry. J. Mater. Chem. A 2015, 3, 21797-21804.

(22) Dan, Y.; Popowski, Y.; Buzhor, M.; Menashe, E.; Rachmani, O.; Amir, E. Covalent surface modification of cellulose-based textiles for oil-water separation applications. Ind. Eng. Chem. Res. 2020, 59, $5456-5465$.

(23) Huang, W.; Tang, X.; Qiu, Z.; Zhu, W.; Wang, Y.; Zhu, Y.-L.; Xiao, Z.; Wang, H.; Liang, D.; Li, J.; Xie, Y. Cellulose-Based Superhydrophobic Surface Decorated with Functional Groups Showing Distinct Wetting Abilities to Manipulate Water Harvesting. ACS Appl. Mater. Interfaces 2020, 12, 40968-40978.

(24) Yao, H.; Lu, X.; Chen, S.; Yu, C.; He, Q. S.; Xin, Z. A Robust Polybenzoxazine/SiO2 Fabric with Superhydrophobicity for HighFlux Oil/Water Separation. Ind. Eng. Chem. Res. 2020, 59, 77877796.
(25) Chen, X.; Wang, B.; Chu, R.; Xing, T.; Chen, G. Fabrication of Robust Water-Repellent Technology on Cotton Fabric via Reaction of Thiol-ene Click Chemistry. Coatings 2020, 10, No. 508.

(26) Xi, G.; Wang, J.; Luo, G.; Zhu, Y.; Fan, W.; Huang, M.; Wang, H.; Liu, X. Healable superhydrophobicity of novel cotton fabrics modified via one-pot mist copolymerization. Cellulose 2016, 23, 915927.

(27) Shang, Q.; Liu, C.; Chen, J.; Yang, X.; Hu, Y.; Hu, L.; Zhou, Y.; Ren, X. Sustainable and Robust Superhydrophobic Cotton Fabrics Coated with Castor Oil-Based Nanocomposites for Effective OilWater Separation. ACS Sustainable Chem. Eng. 2020, 8, 7423-7435.

(28) Lei, M.; Li, Y.; Liu, Y.; Ma, Y.; Cheng, L.; Hu, Y. Effect of Weaving Structures on the Water Wicking-Evaporating Behavior of Woven Fabrics. Polymers 2020, 12, No. 422.

(29) Ravandi, S. A. H.; Mehrara, S.; Sadrjahani, M.; Haghi, A. K. Tunable wicking behavior via titanium oxide embedded in polyacrylonitrile nanofiber strings of yarn. Polym. Bull. 2020, 77, 307-322.

(30) Wang, X.; Yu, P.; Zhang, K.; Wu, M.; Wu, Q.; Liu, J.; Yang, J.; Zhang, J. Superhydrophobic/superoleophilic cotton for efficient oilwater separation based on the combined octadecanoyl chain bonding and polymer grafting via surface-initiated ATRP. ACS Appl. Polym. Mater. 2019, 1, 2875-2882.

(31) Li, T.; Liu, Y.; Wang, Y.; Wang, Y.; Ma, P.; Zhang, S.; Chen, M.; Dong, W. Superhydrophobic Composite Cotton Generated from Raspberry-like Nanoparticles and Their Applications in Oil/Water Separation. Ind. Eng. Chem. Res. 2020, 59, 16305-16311.

(32) Wu, Y.; Zeng, J.; Si, Y.; Chen, M.; Wu, L. Large-Area Preparation of Robust and Transparent Superomniphobic Polymer Films. ACS Nano 2018, 12, 10338-10346.

(33) Zhang, B.; Xu, W. Superhydrophobic, superamphiphobic and SLIPS materials as anti-corrosion and anti-biofouling barriers. New J. Chem. 2021, 15170-15179.

(34) Zhang, B. B.; Duan, J. Z.; Huang, Y. L.; Hou, B. R. Double layered superhydrophobic PDMS-Candle soot coating with durable corrosion resistance and thermal-mechanical robustness. J. Mater. Sci. Technol. 2021, 71, 1-11.

(35) Zhang, B. B.; Xu, W. C.; Zhu, Q. J.; Hou, B. R. Scalable, fluorine free and hot water repelling superhydrophobic and superoleophobic coating based on functionalized Al2O3 nanoparticles. J. Mater. Sci. Technol. 2021, 66, 74-81.

(36) Song, L.; Hu, J.; Huang, X.; Zhong, L.; Pei, Y.; Wu, L.; Zhang, $\mathrm{X}$. Superhydrophobic Self-Healing Coatings Comprised of Hemispherical Particles Arrays Decorated by Fluorocarbon-Coated Nanoscale $\mathrm{Fe} 2 \mathrm{O} 3$ Rods and $\mathrm{SiO} 2$ Particles. ACS Appl. Nano Mater. 2020, 3, 10342-10348. 